Ann. Biol. anim. Bioch. Biophys., 1979, 19 (3 B), 833-836.

\title{
Dérivation de la sécrétion biliaire et activités enzymatiques dans le tissu pancréatique chez le porc
}

\author{
par T. CORRING, J. MOUROT, M. CHARLES *, C. LÉGER ** \\ avec la collaboration technique de Anne-Marie GUEUGNEAU, Georgette FREDOU, A. ROGER et \\ A. VAN HECKE \\ Laboratoire de Physiologie de la Nutrition, I.N.R.A., 78350 jouy-en-Josas \\ * CNRS-CBBM 31, chemin joseph Aiguier 13274 Marseille Cedex 2 \\ ** Station de Recherches sur la Nutrition, I.N.R.A., 78350 Jouy-en-Josas.
}

Summary. Suppression of bile secretion and enzyme activities in pig pancreatic tissue.

The intestinal hydrolysis of dietary triglycerides needs the presence of pancreatic lipase and colipase and of bile salts. However, in in vitro lipolysis there is an opposition between the inhibitory effect of bile salts and the reverse action of colipase. Is this opposition in vivo dependent on a physiological regulation of colipase and bile salt secretions? The experiment reported here aimed to study the effect of long-term (8 days) suppression of bile from the intestinal lumen on colipase and digestive enzyme activities in pig pancreatic issue. Fifteen pigs were fitted with permanent fistulae in the bile duct and the duodenum. In 7 pigs, the collecied bile was returned to the intestine, while in the other 8 pigs an electrolyte solution was introduced into the intestine to replace the bile which had been collected. The results showed no significant differences in lipase, amylase, chymotrypsin and trypsin activities in either group of animals.

La digestion des triglycérides alimentaires dans l'intestin est le résultat de l'action d'une enzyme, la lipase et de son cofacteur, la colipase, toutes deux d'origine pancréatique, sur le substrat convenablement émulsifié par l'action des sels ef phospholipides biliaires. On a pu montrer, in vitro, au cours de ces dernières années (Chapus et al., 1975 ; Borgström, 1977 ; Rietsch et al., 1977 ; Verger, Rietsch et Desnuelle, 1977), le rôle respectif des différents facteurs de la lipolyse. On sait que la lipase est seule responsable de l'acte catalytique et que les sels biliaires sont notamment nécessaires pour stabiliser le substrat sous forme de globules d'émulsion de un à quelques microns de diamètre, permettant d'augmenter la surface de contact huile/eau du contenu digestif. Mais la présence des sels biliaires rend l'interface plus polaire et favorise la désorption de l'enzyme. La colipase intervient alors par sa capacité à se fixer sur les surfaces polaires, pour maintenir la lipase au contact de son substrat et permettre son action.

Le fait important dans ce mécanisme complexe est l'opposition entre l'effet inhibiteur des sels biliaires ef l'effet inverse dû à la colipase. Cet antagonisme au niveau 
moléculaire est-il sous la dépendance d'une régulation physiologique entre sécrétion biliaire et sécrétion colipasique?

Dans le but d'aborder l'étude de l'existence d'une telle régulation, une première éfude a été réalisée chez le porc et elle vise à mesurer le niveau tissulaire pancréatique de la colipase après élimination prolongée de la sécrétion biliaire de la lumière intestinale.

\section{Matériel et méthodes.}

Animaux et régime. - Quinze porcs mâles castrés, de race Large White et de poids vif moyen de $45 \mathrm{~kg}$ en début d'expérience ont été utilisés. Tous les animaux ont été munis de fistules permanentes du bas cholédoque et du duodénum selon la technique décrite dans un précédent travail (Juste, Corring ef Breant, 1979). Le régime alimentaire est identique à celui utilisé lors d'expérimentations précédentes (Corring, Aumaître et Rérat, 1972 ; Juste, Corring et Breant, 1979). Il a été distribué en 2 repas de $800 \mathrm{~g}$ chacun (poids aliment/poids eau : 1/2) par jour.

Schéma expérimental. - Chez 7 porcs, utilisés comme témoins, la bile recueillie 2 fois par jour a été intégralement retournée à l'animal par la fistule duodénale (Juste, Corring et Breant, 1979). Chez les 8 autres porcs, la bile recueillie est éliminée et on a introduit au niveau duodénal une solution d'électrolytes destinée à compenser les pertes dues à la dérivation biliaire.

Après une période de récupération post-opératoire de 8 jours, les animaux ont été soumis au schéma expérimental décrit, durant une semaine. En fin d'expérimentation, les porcs ont été abattus après un jeûne de $22 \mathrm{~h}$.

Dosages. - Immédiatement après abattage, le pancréas entier est prélevé, pesé et broyé à $0^{\circ} \mathrm{C}$ dans de l'eau distillée $(6,5 \mathrm{ml} / \mathrm{g}$ de pancréas frais). Les protéines totales et les activités enzymatiques de la chymotrypsine, de la trypsine, de l'amylase et de la lipase ont été déterminées dans les homogénats de tissu pancréatique selon des techniques identiques à celles utilisées dans de précédentes études sur le suc pancréatique (Corring et Saucier, 1972 ; Corring, 1974). L'activité de la lipase a été mesurée avant et après saturation en colipase de l'échantillon (Mourot et Corring, 1979) ce qui permet l'expression d'une activité résiduelle ou directe et d'une activité potentielle ou totale respectivement. L'activité totale est utilisée pour estimer le degré de saturation de la lipase directe en colipase présente. Par ailleurs, l'activité colipasique tissulaire a été mesurée après inactivation de la lipase à $\mathrm{pH}$ acide (Rathelot et al., 1975) selon une technique décrite précédemment (Mourot et Corring, 1969).

\section{Résultats.}

Le poids relatif du pancréas (par $\mathrm{kg}$ de poids vif), la teneur en protéines totales du tissu pancréatique ef les activités spécifiques enzymatiques (exprimées par $\mathrm{mg}$ de protéines totales) pour les 2 lots d'animaux sont rapportés dans le tableau 1.

On constate quel que soit le critère considéré, qu'il n'existe aucune différence significative entre les données obtenues chez les animaux témoins et les animaux privés de bile dans la lumière intestinale. 
TABLEAU 1

Activités enzymatiques et colipasique dans le tissu pancréatique du porc

après dérivation de la bile (8 jours) de la lumière infestinale.

\begin{tabular}{|c|c|c|c|}
\hline & $\begin{array}{c}\text { Lot expérimental } \\
\text { (1) }\end{array}$ & Lot témoin $\left({ }^{2}\right)$ & $\begin{array}{l}\text { Signification } \\
\text { statistique }\end{array}$ \\
\hline 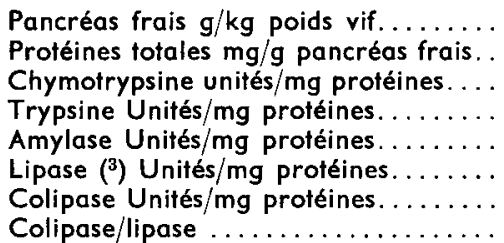 & $\begin{array}{c}1,97 \pm 0,3 \\
169,2 \pm \quad 9,4 \\
12,1 \pm 0,9 \\
2,58 \pm \quad 0,1 \\
3128 \pm 282 \\
29,2 \pm 2,3 \\
33,2 \pm \quad 2,5 \\
1,14 \pm \quad 0,04\end{array}$ & $\begin{array}{c}1,97 \pm 0,2 \\
175,2 \pm 10,7 \\
11,5 \pm 1,1 \\
2,60 \pm 0,06 \\
2768 \pm 148 \\
26,5 \pm 2,4 \\
29,6 \pm 2,3 \\
1,12 \pm 0,03\end{array}$ & $\begin{array}{l}\text { NS } \\
\text { NS } \\
\text { NS } \\
\text { NS } \\
\text { NS } \\
\text { NS } \\
\text { NS } \\
\text { NS }\end{array}$ \\
\hline
\end{tabular}

(1) Animaux ayant subi la dérivation biliaire.

( ${ }^{2}$ ) Animaux dont la bile a été retournée au niveau duodénal.

(3) Lipase potentielle ou totale.

\section{Discussion.}

L'absence de différence entre les valeurs des activités de la colipase obtenues dans les deux groupes d'animaux semble indiquer que l'antagonisme d'action observé in vitro entre colipase et sels biliaires ne se traduit pas in vivo par l'existence d'une régulation physiologique entre l'excrétion biliaire et le niveau de colipase dans le tissu pancréatique.

De plus, il apparaît que l'absence prolongée ( 8 jours) de bile dans la lumière intestinale n'a pas modifié les teneurs tissulaires des enzymes chymotrypsine, trypsine, amylase et lipase.

Les données de la littérature, inexistantes en ce qui concerne la colipase sont très contradictoires en ce qui concerne la relation entre la sécrétion biliaire et la sécrétion pancréatique enzymatique.

En présence de bile ou de sels biliaires dans l'intestin, la sécrétion pancréatique est stimulée (Forell ef al., 1971 ; Konturek et Thor, 1973), inhibée (Thomas et Crider, 1943 ; Malagelada et al., 1972) ou non affectée (lvy ef Lueth, 1927).

Selon Kuroyamagi et Necheles (1962) ef Green et Nasset (1977), chez le rat, l'élimination de bile de la lumière intestinale après obstruction du canal biliaire entraîne une stimulation de la sécrétion pancréatique enzymatique. Ces résultats contradictoires peuvent probablement s'expliquer selon Konturek et Thor (1973), en partie par la méthodologie utilisée pour étudier la sécrétion pancréatique.

Dans l'étude réalisée chez le porc et qui vient d'êłre rapportée, il est important de souligner qu'il n'existe pas de relation entre l'excrétion biliaire ef le niveau tissulaire des enzymes pancréatiques et de la colipase dans les conditions expérimentales choisies. En particulier, la durée prolongée ( 8 jours) de l'élimination de bile de la lumière intestinale pourrait avoir masqué l'apparition d'un phénomène plus rapide et une 
étude réalisée au niveau de l'excrétion pancréatique permettra de vérifier ce point. Par ailleurs, on peut supposer que la quantité de colipase dans le tissu soit telle que l'effet dû à l'élimination de la sécrétion biliaire ne soit pas suffisant pour être expérimentalement mis en évidence.

Commission CNERNA Digestion-Absorption/Association des Physiologistes, Paris 5-6 octobre 1978.

Remerciements. - Ce travail a été réalisé dans le cadre de l'action concertée DGRST 77.7.0472 — Interrelations Aliment - Tube Digestif.

\section{Références}

BORGSTRÖM B., 1977. The action of bile salts and other detergents on pancreatic lipase and the interaction with colipase. Biochim. biophys. Acta, 488, 381-391.

CHAPUS C., SARI H., SEMERIVA M., DESNUELLE P., 1975. Role of colipase in the interfacial adsorption of pancreatic lipase at hydrophilic interfaces. FEBS Letters, 58, 155-158.

CORRING T., 1974. Régulation de la sécrétion pancréatique par rétro-action négative chez le porc. Ann. Biol. anim. Bioch. Biophys., 14, 487-498.

CORRING T., AUMAITRE A., RÉRAT A., 1972. Fisfulation permanente du pancréas exocrine chez le porc. Application : réponse de la sécrétion pancréatique au repas. Ann. Biol, anim. Bioch. Biophys., 12, 109-124.

CORRING T., SAUCIER R., 1972. Sécrétion paneréatique sur porc fistulé. Adaptation à la teneur en protéines du régime. Ann. Biol. anim. Bioch. Biophys., 12, 233-241.

FORELL M. M., OTTE M., KOHL J. H., LEHNERT P., STAHLHEBER H. P., 1971. The influence of bile and pure bile salts in pancreatic secretion in man. Scand. J. Gastroenterol., 6, 262-266.

GREEN G., NASSET E. S., 1977. Effect of bile duct obstruction on pancreatic enzyme secretion and intestinal proteolytic enzyme activity in the rat. Dig. Dis., 22, 437-444.

IVY A. C., LUETH H. C., 1927. Bile stimulation of pancreatic secretion. Proc. Soc. exp. Biol. Med., 24, 837-845.

JUSTE C., CORRING T., BREANT Ph., 1979. Excrétion biliaire chez le porc : niveau et réponse au repas. Ann. Biol. anim. Bioch. Biophys., 19, 79-90.

KONTUREK S. J., THOR P., 1973. Effect of diversion and replacement of bile on pancreatic secretion. Dig. Dis. 18, 971-977.

KUROYAMAGI Y., NECHELES H., 1962. Pancreatic secretion in the rat : effect of bile stasis and of bile salt. Am. J. Physiol., 203, 60-62.

MALAGELADA J. R., GO V. L. W., DIMAGNO E. P., SUMMERSKILL W. H. J., 1972. Interactions of bile acid and digestive products on the release of cholecystokinin pancreozymin in man. J. clin. Invest., 51, 59a.

MOUROT J., CORRING T., 1979. Adaptation of the lipase-colipase system to diefary lipid content in pig pancreatic tissue. Ann. Biol. anim. Bioch. Biophys., 19, 119-124.

RATHELOT J., JULIEN R., COEROLI C., CANIONI P., SARDA L., 1975. Studies on the effect of bile salt and colipase on enzymatic lipolysis. Improved method for the defermination of pancreatic lipase and colipase. Biochimie, 57, 1117-1122.

RIETSCH J., PATTUS F., DESNUELLE P., VERGER R., 1977. Further studies of mode of action of lipolytic enzymes. J. biol. Chem., 252, 4313-4318.

THOMAS E. J., CRIDER J. C., 1943. The effect of bile in the intestine on the secretion of pancreatic juice. Am. J. Physiol., 138, 548-552.

VERGER R., RIETSCH J., DESNUELLE P., 1977. Effects of colipase on hydrolysis of monomolecular films by lipase. J. biol. Chem., 252, 4319-4325. 